\title{
Being accurate about verbal credibility assessment
}

\author{
Bennett Kleinberg ${ }^{1,2}$, Arnoud Arntz ${ }^{2}$, Bruno Verschuere ${ }^{2}$ \\ ${ }^{1}$ Department of Security and Crime Science, University College London, UK \\ ${ }^{2}$ Department of Psychology, University of Amsterdam, The Netherlands
}

\begin{abstract}
Purpose: Verbal credibility assessments examine language to discern lie from the truth. These tests are used for the scientific study of the language of lies in US Presidential candidates and fraudulent scientists, but also in criminal proceedings for evaluating allegations of child sexual abuse. The dominant approach in psycholegal deception research to date (used in $81 \%$ of recent studies that report on accuracy) to estimate the accuracy of a method is to find the optimal statistical separation between lies and truths in a single dataset. However, this method lacks safeguards against accuracy overestimation.

Method \& Results: A simulation study and empirical data show that this procedure produces overoptimistic accuracy rates that, especially for small sample size studies typical of this field, yield misleading conclusions up to the point that a non-diagnostic tool can be shown to be a valid one. Cross-validation is a cheap and easy remedy to this problem.

Conclusions: We propose a set of guidelines to make the psycholegal research meet highest standards and encourage researchers to be more accurate about accuracy and to conduct proper validation when reporting accuracy rates.
\end{abstract}

Transparency statement:

The data and code to reproduce the analysis are publicly available on the Open Science Framework at https://osf.io/2des5/. 


\section{TECHNICAL NOTE}

\section{Being accurate about statement credibility assessment}

A linguistic analysis of papers written by former social psychology Professor Diederik Stapel showed that his fraudulent papers expressed greater certainty than his articles that were based on actual data (Markowitz \& Hancock, 2014). Analyzing fact-checked statements from the 2016 US presidential elections showed that using cognitive operators (e.g., words such as "cause" or "know") were indicators of Hillary Clinton and Donald Trump's lying (Bond et al., 2017). Verbal credibility assessment is not only used to expose deceit in fraudulent scientists and presidential candidates but is also widely applied in legal proceedings. When no physical evidence is available courts have to rely heavily on statement credibility analysis.

The recent case of allegations of sexual misconduct against US supreme court nominee Brett Kavanaugh illustrates the challenge. Kavanaugh was accused of a sexual assault more than 30 years before his nomination (Brown, 2018; Kelly \& Estepa, 2018). In the absence of any other evidence, a Judiciary Committee hearing revolved exclusively around statements made by Kavanaugh and his accuser, thereby requiring the committee to base its decision on an assessment of the credibility of the statements made.

Several US federal agencies including the FBI, Army Military Intelligence, and the US Immigration and Naturalization Service have been trained in Scientific Content Analysis (SCAN, see http://www.lsiscan.com/id29.htm). Courts in Germany, Sweden, the UK and the Netherlands have accepted Criteria-based Content Analysis (CBCA) as a means to determine the veracity of allegations of child sexual abuse (Oberlader et al., 2016), and a promising new tool for airport security screening is predominantly based on verbal indicators of deception (Ormerod \& Dando, 2015). While different in the exact scoring methods applied, all these statement credibility assessment methods share a standard procedure: suspects or witnesses are asked to provide a statement about an (alleged) event, which is the transcribed and analysed by experts on a range of dimensions (e.g., the level of detail, the plausibility of the statement). But how accurate are such verbal credibility assessment tools?

The typical way in which psycholegal deception researchers answer that question is by building a dataset consisting of statements that are known to be truthful or deceptive (e.g., by instructing participants to tell a lie or the truth), extracting a set of verbal indicators from each statement (e.g., its logical structure, detailedness), and use linear discriminant analysis - a statistical technique that will provide the optimal function of verbal indicators to discern lie from truth (Fisher, 1936). While there are diverse methods to assess the "goodness" of these methods, a review of primary studies included in the most recent meta-analyses in the field suggests that there is a clear preference for one specific statistical procedure. When researchers reported an accuracy of their approach (i.e. the percentage of truthful and deceptive statements identified as such), in $77 \%$ of the cases they applied a classification algorithm (e.g., linear discriminant analysis but it applies to practically all classification methods including, for example, logistic regression) that built on the same dataset that it is tested on. ${ }^{1}$ This figure has not changed; papers published between 2010-2017 show that 81\% make use of that statistical procedure that we call the "training set optimisation technique". By capitalising on idiosyncrasies of the dataset, this procedure diminishes its vital goal:

\footnotetext{
${ }^{1}$ See Appendix A.
} 
assessing the 'true' accuracy of the classification if it were to make a credibility prediction for a novel, out-ofsample set of statements (see also the Levine, Blair, \& Carpenter, 2017).

\section{Method and Results}

We simulated data for a set of verbal predictors that are either unrelated (correlation of $r=0.0$ ) or weakly related $(r=0.1)$ to a binary outcome (lie vs truth). ${ }^{2}$ The latter was chosen to reflect the small effect sizes that are common in verbal deception research (e.g., Amado, Arce, Fariña, \& Vilariño, 2016; DePaulo et al., 2003; Vrij, Granhag, $\&$ Porter, 2010; the average for the 19 CBCA criteria is $d=.25$ or $r=.12$ in Amado et al., 2016).

\section{The current practice}

Simulation procedure: We simulated data for a sample of $n=1,000$ with 8 (as with a popular verbal approach used in research, Reality Monitoring, Hauch, Sporer, Masip, \& Blandón-Gitlin, 2017), 12 (as for a popular verbal approach used in practice, SCAN, Bogaard, Meijer, Vrij, Broers, \& Merckelbach, 2014), or 19 (as with CBCA, Hauch et al., 2017) predictors. The correlation between the predictors and the binary outcome was held constant at either $r=0.0, r=0.1$, or $r=0.124$. We iteratively simulated data for sample sizes of $n=40$ up until $n=1,000$ in steps of 10. For each step, we ran 100 simulations and averaged the classification accuracies. For each sample size, we calculated the accuracy obtained without cross-validation (= training set optimisation) and with leaveone-out cross-validation. Also, for each predictive model, we tested the accuracy on a second simulated set of observations (test set) of the same size (i.e., of the training set was $n=40$, the test set also was $n=40$ ). The class membership of the binary outcome (i.e., proxies for truthful vs deceptive) was 50/50, and the priors in the latent discriminant analysis were set accordingly. The simulations were conducted using the caret (Kuhn, 2017) and MASS (Venables, Ripley, \& Venables, 2002) packages in R (R Core Team, 2016). The plots were created using ggplot2 (Wickham, 2009).

Findings: Figure 1 shows the volatility in the accuracy as estimated by latent discriminant analysis when using 8 , 12 , or 19 predictors. For the sample sizes typical for the field $\left(M_{n}=61\right.$, range ${ }_{n}=10$ to 240 in the most recent meta-analyses, Oberlader et al., 2016; Vrij, Fisher, \& Blank, 2015) the accuracy estimates are unreliable and display a highly volatile pattern. The weaker the diagnostic value of the verbal indicators and the more indicators used, the more problematic the erroneous accuracy estimates. In a sample of 40 participants, a completely undiagnostic verbal tool $(r=0.0)$ with 19 indicators can be estimated to have up to $84 \%$ accuracy. In sum, the dominant practice of using linear discriminant analysis on a single dataset provides highly inaccurate and overly optimistic accuracy estimates.

\footnotetext{
${ }^{2}$ The code for the simulation studies and the resulting reproducible data are available at https://osf.io/2des5/files/
} 


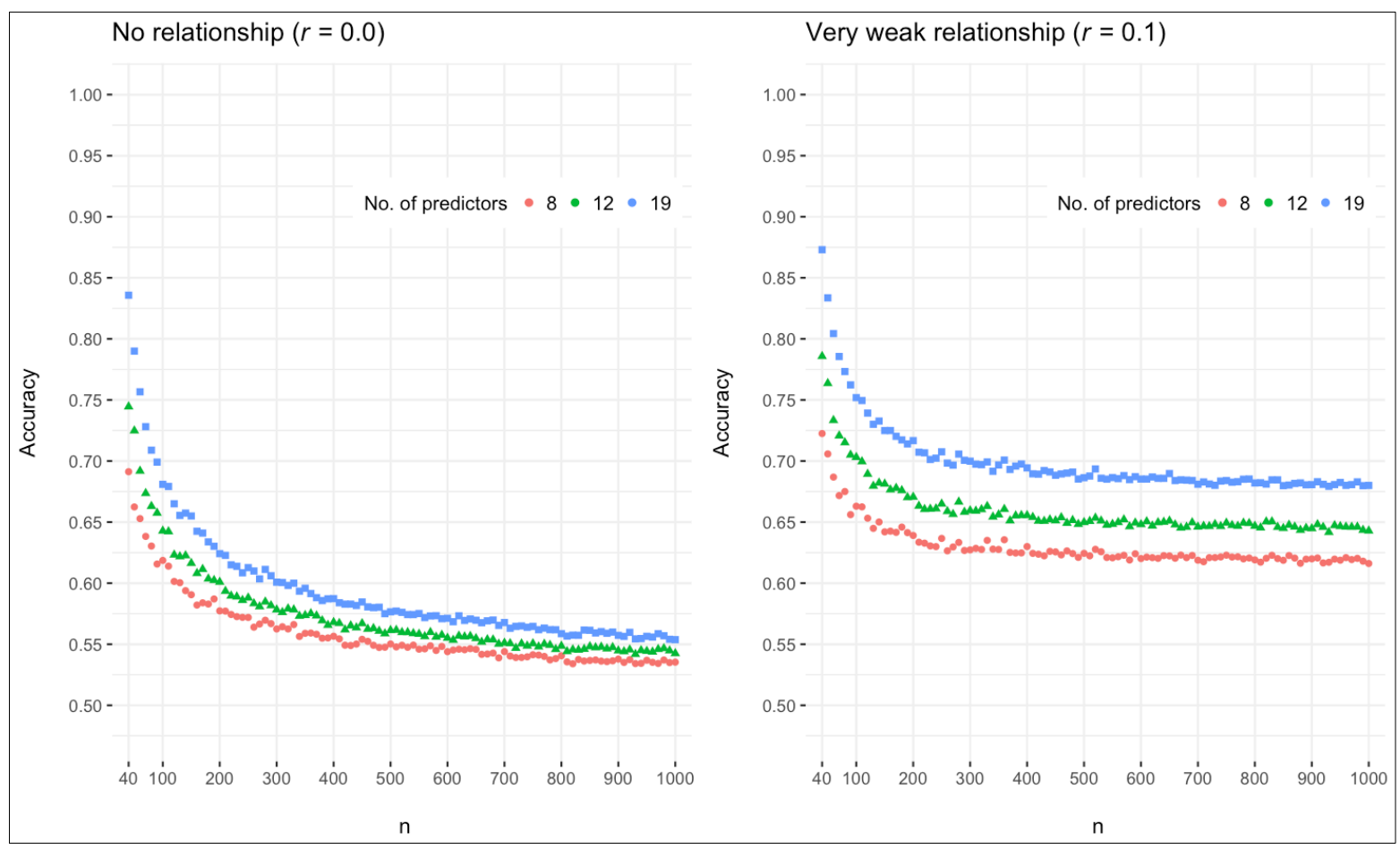

Figure 1. Accuracies for Latent Discriminant Analysis without cross-validation on simulated data for 8, 12 and 19 predictors with increasing $n$.

\section{Fixing the problem}

The desirable evaluation of the predictive accuracy of a classification algorithm occurs when the algorithm is built on one dataset and tested on an entirely new dataset. However, is it always necessary to collect new data? Especially when high-quality data are hard to obtain (e.g., allegations of child sexual abuse; the primary usage of the CBCA) or labor-intensive (e.g., several trained human coders rate a large number of statements on a number of verbal criteria) it would be desirable to have a precise accuracy estimate derived from a single dataset. A solution, then, is to treat the individual dataset as if it were multiple datasets (Yarkoni \& Westfall, 2017). Known as cross-validation, this procedure handles a part of the dataset as the model building dataset (or training set), and another part to assess its performance (test set; Arlot \& Celisse, 2010). Analogous to the training-vs-test set terminology, the training set optimisation technique reports accuracies of the training set only, thereby lacking the critical evaluation of the predictions.

Procedure: To examine how the current practice - the training set optimization technique - compares to crossvalidation and independent validation, we simulated data for 19 predictors (as in CBCA) with a weak individual predictor-outcome relationship ( $r=0.124$, converted from Cohen's $d=0.27$, the average effect size for CBCA criteria in a recent meta-analysis; Amado, Arce, Fariña, \& Vilariño, 2016). We calculated the prediction accuracies - for increasing sample sizes - obtained from linear discriminant analysis using both the training set optimisation technique and leave-one-out cross-validation. The latter builds the algorithmic prediction model on $n-1$ data points and tests it iteratively on the single held-out observation. That procedure recycles data and ensures that each data point is used for validation exactly once. Both procedures resulted in a predictive model (i.e. empirically determined linear combinations of the predictors that separate the data into two classes - deceptive and truthful) that was then additionally validated on a novel, also simulated, test set of the same size. 
Findings: Figure 2 shows the differences between the accuracies yielded on the training set with the current practice of training set optimisation and our recommended leave-one-out cross-validation, and the independent test set. We adopted the points-of-stability procedure (Schönbrodt \& Perugini, 2013) to evaluate at which sample size, the fluctuation is deemed practically irrelevant. We defined a stability corridor [ $+0.05 ;-0.05]$, that allowed us to find the point-of-stability in $n$ after which the accuracy difference compared to the test set evaluation does not leave the stability corridor. Figure 2 allows for the following conclusions:

- When relying on the dominant training set optimisation, the sample size needs to be substantially larger than is typical in the psycholegal literature $(n>320)$ to eliminate accuracy overestimations.

- The overestimation of the accuracy achieved with the training set optimisation technique is substantial. This is especially the case for sample sizes commonly used in verbal credibility assessment research (40 $<=n<=240$ ). For these sample sizes, the overestimation is on average 12 percentage points in accuracy (range: 6 to 29). For previous reports that lack cross-validation, this graph can be used as a means to estimate the magnitude of inflation in the reported accuracy. For example, for a study with 80 participants without cross-validation, the accuracy should be corrected downwards with at least 12 accuracy points (e.g., reported accuracy $=75 \%$; corrected estimate $=63 \%$ ).

- Cross-validation safeguards against accuracy overestimation. Cross-validated predictive models fare consistently well when validated on a test set; the accuracy difference never exceeded $5 \%$ even for small sample sizes.

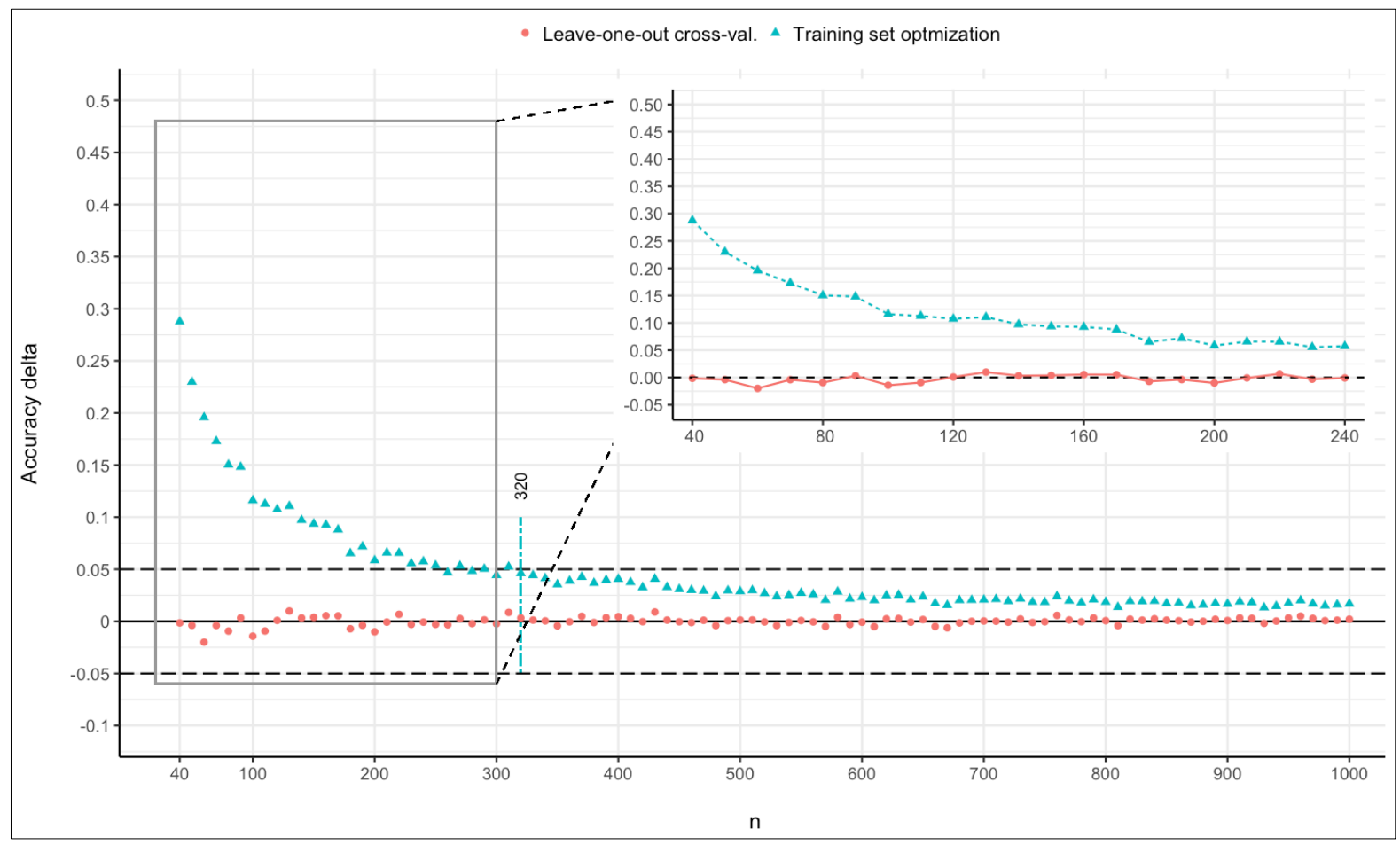

Figure 2. Accuracy differences between traditional training set optimisation and leave-one-out cross-validation compared to independent test set validation. The dashed horizontal grey lines indicate the upper and lower boundary of the $[-0.05 ;+0.05]$ stability corridor. The vertical coloured line indicates the sample size points-of-stability for the training set optimisation technique. Inset plot: accuracy difference scores zoomed in for sample size between 40 and 240 . 


\section{The importance of cross-validation: Illustration with real verbal credibility assessment data}

To illustrate the importance of validation outside of the simulation scenario, we obtained the raw data from recently published verbal deception detection studies. Two datasets were about truthful and deceptive statements of someone's recent negative autobiographical event, manually annotated with SCAN (Bogaard, Meijer, \& Vrij, 2014; Bogaard, Meijer, Vrij, \& Merckelbach, 2016) - a popular yet in the academic community heavily criticized tool for having low reliability and validity (Bogaard et al., 2016; Vanderhallen, Jaspaert, \& Vervaeke, 2016; Vrij, 2015). Table 1 shows that linear discriminant analysis on the original dataset without cross-validation (= current practice) misleadingly would suggest SCAN to be able to discern a lie from the truth. As shown above, crossvalidation can protect from accuracy inflation. With cross-validation, it becomes clear that SCAN did not perform better than chance. It is troubling that the dominant practice would present the heavily criticised and nonsubstantiated tool SCAN (Bogaard et al., 2016; Vanderhallen et al., 2016; Vrij, 2015) as a valid method (i.e. better than chance accuracy) to classify lies and truths.

Table 1. Illustration how the dominant practice (linear discriminant analysis with training set optimisation) can lead to an erroneous conclusion.

\begin{tabular}{ccc}
\hline & $\begin{array}{c}\text { Training set optimisation (current } \\
\text { practice) }\end{array}$ & $\begin{array}{c}\text { Leave-one-out cross-validation } \\
\text { (recommended practice) }\end{array}$ \\
\hline \multirow{2}{*}{ Accuracy estimate } & $61.54 \%$ & $51.28 \%$ \\
& {$[54.98-67.80]$} & {$[44.68-57.85]$} \\
Conclusion & "Significantly & "No better than chance \\
& classification."
\end{tabular}

\section{Discussion}

Reviewing the literature on verbal credibility assessment, we note that the vast majority of studies that report accuracy rates try to find the optimal statistical separation between deceptive and truthful statements through verbal criteria within a single dataset. Through simulations, we show that this technique leads to imprecise accuracy estimates for the sample sizes that are typical for the verbal deception detection field. Accuracy estimates are systematically overestimated for $n<320$. Our simulations suggest that the accuracies reported in the majority of published verbal deception detection research may need to be corrected downwards with up to 12 accuracy points due to a suboptimal statistical classification procedure. Such a correction is substantial considering that the average accuracy rates of verbal deception research as a whole only exceed the random guessing baseline by about 20 accuracy points (Hauch et al., 2017; Vrij et al., 2015).

\section{Suggestions for remedy}

Our proposed solution is three-fold (Table 2). First, at the minimum, we recommend using cross-validation for all prediction algorithms. Cross-validation comes at practically no cost, is provided by all statistical software packages (e.g., SPSS, R, JASP), and ensures a leap in accuracy precision. Note that cross-validation is standard practice in computational disciplines (using machine learning). It may not be new to deception research either (see, e.g., Köhnken, Schimossek, Aschermann, \& Höfer, 1995) but remains infrequently used as of to date. ${ }^{3}$ It is

\footnotetext{
${ }^{3}$ Note that we are not introducing cross-validation as a new concept, nor are we the first to advocate the use of cross-validation as a safeguard against inflated accuracy estimated. However, given the far-reaching
} 
worthwhile pointing out that cross-validation comes in many fashions and thereby leaves the exact choices to the researcher (e.g. one could use leave-one-out, split-half, holdout, or re-usable holdout cross-validation, to name but a few; Arlot \& Celisse, 2010; Dwork et al., 2015; Kim, 2009). To avoid that cross-validation is tried repeatedly until favourable results are obtained - analogous to $p$-hacking in behavioural research (Gelman \& Loken, 2013) one may pre-register the cross-validation procedures (i.e. determining the procedure beforehand, making a public pre-registration of that procedure, and reporting the findings of that procedure; Savi, Ruijs, Maris, \& van der Maas, 2018).

Second, even preferable to cross-validation is testing the prediction algorithm on a new sample. Since cross-validation still relies on just one sample and because the essential test of a method is how well it performs on "unseen" data, researchers would want to assess a classifier algorithm derived from one data collection moment (e.g. one experiment) on freshly collected data (e.g. a second, identical experiment). Early findings on such independent sample validation in verbal deception research suggest that classification algorithms are less robust against sample variations than expected (Kleinberg, Toolen, Vrij, Arntz, \& Verschuere, 2018). This independent sample validation would at the same time provide for the much needed direct replications of verbal deception studies (Simons, 2014; for an exception see: Kleinberg, Warmelink, Arntz, \& Verschuere, 2018). Independent sample validation ideally implies that the classifier used is pre-registered. However, because it is not always feasible to re-run an experiment, a fast and easy alternative is to share the classifier algorithm derived from a study with the community. Each classifier can be stored in a sharable data file using statistical software. By making the classifier available, other researchers can test how well it performs on their dataset.

Third, increasing sample sizes. Such a call for larger sample sizes has repeatedly been made (Wagenmakers et al. 2011, Yarkoni \& Westfall, 2017), but we think that our current demonstration may help convince verbal deception researchers of its importance. The sample sizes of verbal deception research are small, and the sharing of data is still scant. This leaves a void of large datasets that are needed to assess the methods of the field reliably. We strongly encourage the research community to conduct studies with larger sample sizes. With a move towards better research practices, in particular in psychological research, we agree with Yarkoni and Westfall that "[in] many cases there is a serious debate to be had about whether it is scientifically useful to conduct small-sample research at all" (Yarkoni \& Westfall, 2017, p. 1110).

implications of verbal deception research in practice and the lack of any cross-validation in the majority of studies, we feel that the field as a whole would benefit from a gentle reminder to use better methods to estimate the goodness of the methods used. 
Table 2. Suggestions for the improvement of the accuracy estimation in the predictive analysis in verbal credibility assessment research.

\begin{tabular}{|c|c|c|c|c|}
\hline Remedy & & Key Advantage & Key challenge & Open Science safeguard \\
\hline $\begin{array}{c}\text { Validation on an } \\
\text { independent sample }\end{array}$ & - & $\begin{array}{l}\text { Allows for robust claims } \\
\text { regarding the } \\
\text { generalizability of findings }\end{array}$ & $\begin{array}{l}\text { Resource intensive } \\
\text { (new data } \\
\text { collection) }\end{array}$ & $\begin{array}{ll}- & \text { Pre-registration of the } \\
\text { classification algorithm } \\
\text { - } & \text { Sharing classification } \\
\text { algorithm as a data file }\end{array}$ \\
\hline Cross-validation & - & $\begin{array}{l}\text { Easy to implement (no } \\
\text { new data collection } \\
\text { needed, often default } \\
\text { setting in statistical } \\
\text { software) }\end{array}$ & $\begin{array}{l}\text { Might still capitalise } \\
\text { on idiosyncrasies of } \\
\text { the sample }\end{array}$ & $\begin{array}{l}\text { Pre-registration of } \\
\text { cross-validation } \\
\text { procedure }\end{array}$ \\
\hline Larger sample sizes & - & $\begin{array}{l}\text { Solidifies conclusions on } \\
\text { statistical inferences and } \\
\text { prediction metrics }\end{array}$ & Resource intensive & $\begin{array}{ll}\text { - } & \text { Preregistration of } \\
\text { sample size justification } \\
\text { - } \quad \text { Open sharing of data }\end{array}$ \\
\hline
\end{tabular}

\section{An optimistic outlook}

Verbal credibility assessment and hence its scholarly backcloth of verbal deception research remains of vital importance in legal settings, and it is not foreseeable that this will change in the nearby future. Therefore, it should be in the interest of the general public, the scholarly community and practitioners that the reporting of the goodness of the methods that can have dramatic consequences meet the highest standards. The highest standards have changed over the past years, and we observed that many studies do not meet these criteria. Because deception detection is a topic too important not to be pursued, we argue that as a research community we should strive to be at the forefront of transparent and careful research practices. The psycholegal deception research community has recently started a discussion about more research transparency (Nahari et al., 2019), and we hope that this paper adds to the debate and encourages more precise statistical procedures. Given its broad applications and farreaching implications, it is imperative that verbal credibility research becomes more accurate about its accuracy. 


\section{References}

Amado, B. G., Arce, R., Fariña, F., \& Vilariño, M. (2016). Criteria-Based Content Analysis (CBCA) reality criteria in adults: A meta-analytic review. International Journal of Clinical and Health Psychology, 16(2), 201-210. https://doi.org/10.1016/j.ijchp.2016.01.002

Arlot, S., \& Celisse, A. (2010). A survey of cross-validation procedures for model selection. Statistics Surveys, 4(0), 40-79. https://doi.org/10.1214/09-SS054

Bogaard, G., Meijer, E. H., \& Vrij, A. (2014). Using an Example Statement Increases Information but Does Not Increase Accuracy of CBCA, RM, and SCAN: Using an example statement with truth tellers and liars. Journal of Investigative Psychology and Offender Profiling, 11(2), 151-163.

https://doi.org/10.1002/jip.1409

Bogaard, G., Meijer, E. H., Vrij, A., Broers, N. J., \& Merckelbach, H. (2014). SCAN is largely driven by 12 criteria: results from sexual abuse statements. Psychology, Crime \& Law, 20(5), 430-449. https://doi.org/10.1080/1068316X.2013.793338

Bogaard, G., Meijer, E. H., Vrij, A., \& Merckelbach, H. (2016). Scientific Content Analysis (SCAN) Cannot Distinguish Between Truthful and Fabricated Accounts of a Negative Event. Frontiers in Psychology, 7. https://doi.org/10.3389/fpsyg.2016.00243

Bond, G. D., Holman, R. D., Eggert, J.-A. L., Speller, L. F., Garcia, O. N., Mejia, S. C., ... Rustige, R. (2017). 'Lyin” Ted', “Crooked Hillary", and "Deceptive Donald": Language of lies in the 2016 US presidential debates: Language of lies in debates.' Applied Cognitive Psychology, 31(6), 668-677. https://doi.org/10.1002/acp.3376

Brown, E. (2018). California professor, writer of confidential Brett Kavanaugh letter, speaks out about her allegation of sexual assault. Retrieved February 10, 2019, from https:/www.washingtonpost.com/investigations/california-professor-writer-of-confidential-brettkavanaugh-letter-speaks-out-about-her-allegation-of-sexual-assault/2018/09/16/46982194-b846-11e894eb-3bd52dfe917b_story.html

DePaulo, B. M., Lindsay, J. J., Malone, B. E., Muhlenbruck, L., Charlton, K., \& Cooper, H. (2003). Cues to deception. Psychological Bulletin, 129(1), 74-118. https://doi.org/10.1037/0033-2909.129.1.74

Dwork, C., Feldman, V., Hardt, M., Pitassi, T., Reingold, O., \& Roth, A. (2015). The reusable holdout: Preserving validity in adaptive data analysis. Science, 349(6248), 636-638. https://doi.org/10.1126/science.aaa9375 
Fisher, R. A. (1936). The use of multiple measurements in taxonomic problems. Annals of Eugenics, 7(2), 179188. https://doi.org/10.1111/j.1469-1809.1936.tb02137.x

Gelman, A., \& Loken, E. (2013). The garden of forking paths: Why multiple comparisons can be a problem, even when there is no "fishing expedition" or "p-hacking" and the research hypothesis was posited ahead of time. Retrieved January 19, 2018, from http://www.stat.columbia.edu/ gelman/research/unpublished/p_hacking.pdf

Hauch, V., Blandón-Gitlin, I., Masip, J., \& Sporer, S. L. (2015). Are computers effective lie detectors? A metaanalysis of linguistic cues to deception. Personality and Social Psychology Review, 19(4), 307-342. Retrieved from http://journals.sagepub.com/doi/abs/10.1177/1088868314556539

Hauch, V., Sporer, S. L., Masip, J., \& Blandón-Gitlin, I. (2017). Can credibility criteria be assessed reliably? A meta-analysis of criteria-based content analysis. Psychological Assessment, 29(6), 819-834. https://doi.org/10.1037/pas0000426

Kelly, E., \& Estepa, J. (2018). Brett Kavanaugh: Timeline of allegations against SCOTUS nominee. Retrieved February 10, 2019, from http://www.usatoday.com/story/news/politics/onpolitics/2018/09/24/brettkavanaugh-allegations-timeline-supreme-court/1408073002/

Kim, J.-H. (2009). Estimating classification error rate: Repeated cross-validation, repeated hold-out and bootstrap. Computational Statistics \& Data Analysis, 53(11), 3735-3745. https://doi.org/10.1016/j.csda.2009.04.009

Kleinberg, B., Toolen, Y. van der, Vrij, A., Arntz, A., \& Verschuere, B. (2018). Automated verbal credibility assessment of intentions: The model statement technique and predictive modeling. Applied Cognitive Psychology, 32(3), 354-366. https://doi.org/10.1002/acp.3407

Kleinberg, B., Warmelink, L., Arntz, A., \& Verschuere, B. (2018). The first direct replication on using verbal credibility assessment for the detection of deceptive intentions. Applied Cognitive Psychology. https://doi.org/10.1002/acp.3439

Köhnken, G., Schimossek, E., Aschermann, E., \& Höfer, E. (1995). The cognitive interview and the assessment of the credibility of adults' statements. Journal of Applied Psychology, 80(6), 671.

Kuhn, M. (2017). caret: Classification and Regression Training (Version R package version 6.0-76). Retrieved from https://CRAN.R-project.org/package $=$ caret 
Levine, T. R., Blair, J. P., \& Carpenter, C. J. (2017). A critical look at meta-analytic evidence for the cognitive approach to lie detection: A re-examination of Vrij, Fisher, and Blank (2017). Legal and Criminological Psychology. https://doi.org/10.1111/lcrp.12115

Markowitz, D. M., \& Hancock, J. T. (2014). Linguistic Traces of a Scientific Fraud: The Case of Diederik Stapel. PLoS ONE, 9(8), e105937. https://doi.org/10.1371/journal.pone.0105937

Nahari, G., Ashkenazi, T., Fisher, R. P., Granhag, P.-A., Hershkowitz, I., Masip, J., ... Vrij, A. (2019). 'Language of lies': Urgent issues and prospects in verbal lie detection research. Legal and Criminological Psychology, 24(1), 1-23. https://doi.org/10.1111/lcrp.12148

Oberlader, V. A., Naefgen, C., Koppehele-Goseel, J., Quinten, L., Banse, R., \& Schmidt, A. F. (2016). Validity of Content-Based Techniques to Distinguish True and Fabricated Statements: A Meta-Analysis. Law and Human Behavior, 40(4), 440-457.

Ormerod, T. C., \& Dando, C. J. (2015). Finding a needle in a haystack: Toward a psychologically informed method for aviation security screening. Journal of Experimental Psychology: General, 144(1), 76-84. https://doi.org/10.1037/xge0000030

R Core Team. (2016). R: A language and environment for statistical computing. Vienna, Austria: R Foundation for Statistical Computing. Retrieved from https://www.R-project.org/

Savi, A. O., Ruijs, N. M., Maris, G. K. J., \& van der Maas, H. L. J. (2018). Delaying access to a problemskipping option increases effortful practice: Application of an A/B test in large-scale online learning. Computers \& Education, 119, 84-94. https://doi.org/10.1016/j.compedu.2017.12.008

Schönbrodt, F. D., \& Perugini, M. (2013). At what sample size do correlations stabilize? Journal of Research in Personality, 47(5), 609-612. https://doi.org/10.1016/j.jrp.2013.05.009

Simons, D. J. (2014). The Value of Direct Replication. Perspectives on Psychological Science, 9(1), 76-80. https://doi.org/10.1177/1745691613514755

Vanderhallen, M., Jaspaert, E., \& Vervaeke, G. (2016). SCAN as an investigative tool. Police Practice and Research, 17(3), 279-293. https://doi.org/10.1080/15614263.2015.1008479

Venables, W. N., Ripley, B. D., \& Venables, W. N. (2002). Modern applied statistics with S (4th ed). New York: Springer.

Vrij, A., Granhag, P. A., \& Porter, S. (2010). Pitfalls and Opportunities in Nonverbal and Verbal Lie Detection. Psychological Science in the Public Interest, 11(3), 89-121.

https://doi.org/10.1177/1529100610390861 
Vrij, Aldert. (2015). Verbal Lie Detection tools: Statement validity analysis, reality monitoring and scientific content analysis. In Detecting deception: Current challenges and cognitive approaches (1st ed., pp. 335). John Wiley \& Sons, Ltd. Retrieved from https://books.google.nl/books?hl=en\&lr=\&id=4brlBQAAQBAJ\&oi=fnd\&pg=RA1PA3\&dq $=$ Verbal+Lie+Detection+tools:+Statement+validity+analysis,,+ reality+monitoring + and + scient ific + content + analysis\&ots $=4$ sFTBKx24S\&sig $=51$ A 5 qnbszpbpaGcYokvw8n37ekw

Vrij, Aldert, Fisher, R. P., \& Blank, H. (2015). A cognitive approach to lie detection: A meta-analysis. Legal and Criminological Psychology, 22(1), 1-21. https://doi.org/10.1111/lcrp.12088

Wickham, H. (2009). Ggplot2: elegant graphics for data analysis. New York: Springer.

Yarkoni, T., \& Westfall, J. (2017). Choosing Prediction Over Explanation in Psychology: Lessons From Machine Learning. Perspectives on Psychological Science, 12(6), 1100-1122. https://doi.org/10.1177/1745691617693393 


\section{Appendix A}

\section{Literature review}

To provide a contemporary overview of the state of classification in human coded verbal deception detection, we reviewed all published papers used in the most recent meta-analyses in the field (Hauch, Blandón-Gitlin, Masip, \& Sporer, 2015; Hauch et al., 2017; Oberlader et al., 2016; Vrij et al., 2015) that reported accuracy rates, used verbal indicators to discriminate between truthful and deceptive statements, and relied on experimental data collection. Of these 169 papers, 56 reported classification analyses - 41 of which (73\%) did not use some (cross-)validation.

The meta-analyses do not include the most recent work on the topic. To explore whether research practices have changed more recently, we also inspected all recent (last five years; 2012-2017), English, peerreviewed papers on human coded verbal credibility assessment. We searched the Web of Science database on the search terms (see Hauch et al., 2017) "Criteria-based content analysis", "CBCA", "reality monitoring”, "RM”, "scientific content analysis", "SCAN", "statement validity assessment", "SVA", "validity checklist" (connected by OR) combined with "psychology of evidence", "statement analysis", "credibility”, "credibility judgment”, "deception" (connected by OR). After excluding overlapping papers already contained in the three metaanalyses, this resulted in an additional 20 papers of which 9 reported classification analyses - 8 of which (89\%) did not use (cross-) validation. The detailed list of all included papers and their annotation is available at https://osf.io/9psek/. 